\title{
ESTUDIO EMPÍRICO SOBRE RESPONSABILIDAD PENAL Y TDAH EN ITALIA
}

\author{
Alberto Pintado Alcázar \\ Profesor Asociado de Derecho Penal y Criminología \\ Universidad de Murcia \\ E-mail: alberto.pintado@um.es
}

\begin{abstract}
RESUMEN: Siempre se ha intentado buscar una explicación veraz que determine el motivo por el cual determinadas personas se convierten en delincuentes. En este sentido, diversas ciencias de conocimiento se han adentrado en diferentes contextos situacionales para intentar esclarecer dicha cuestión.

Actualmente, el Trastorno por Déficit de Atención e Hiperactividad ha sido detectado en una pluralidad de acciones delictivas, con independencia del sujeto dentro de la pareja penal que lo padeciera. No obstante, a pesar de la aparición de este trastorno en sujetos delincuentes, tras el estudio de las diversas sentencias condenatorias encontradas, se ha conseguido determinar que no existe una correlación entre el TDAH y la violencia.
\end{abstract}

PALABRAS CLAVE: TDAH, delincuencia, jurisprudencia, Derecho Penal, Italia.

ABSTRACT: We have always tried to find a truthful explanation to determine the reason why certain people become criminals. In this sense, cognitive science has gone into depth in diverse situational contexts to try to clarify this issue.

At present, Attention Deficit Hyperactivity Disorder has been detected in a variety of criminal actions, independently of the subject within the criminal couple who suffered it. However, despite the appearance of this disorder in criminal subjects, after studying the various condemnatory sentences found, it has been determined that there is no correlation between ADHD and criminal violence.

KEYWORDS: ADHA, Delinquency, Jurisprudence, Criminal Law, Italy.

SUMARIO: I. Fijación de la cuestión. II. Estudio de casos. 1. Situación geográfica. 2. Circunstancias personales. 2.1. Edad de la pareja penal. 2.2. Sexo de los implicados. 3. Características penales. 3.1. Delitos cometidos. 3.2. Circunstancias modificativas de la responsabilidad penal. III. Conclusiones. IV. Jurisprudencia. V. Bibliografia. 


\section{I.- FIJACIÓN DE LA CUESTIÓN}

En primer lugar, cabe destacar que la traducción del Trastorno del Déficit de Atención e Hiperactividad —en adelante, TDAH - en italiano recibe el nombre de Disturbo da Deficit dell'Attenzione ed Iperattività (DDAI). Fue a partir de las investigaciones llevadas a cabo por Chess cuando se comenzó a conocer a este disturbio como «trastorno hipercinético» ${ }^{12}$. Posteriormente, dicho término sería adaptado por el DSM-II, introduciendo a finales de la década de los sesenta la expresión «reacción hipercinética de la niñez» ${ }^{13}$, haciendo referencia a aquellos niños nerviosos, rebeldes e incontrolables.

Así, se considera que dicha expresión se ha ido modificando a lo largo del tiempo, pasando a ser considerada como un daño cerebral mínimo; pero, posteriormente, «en ausencia de una lesión cerebral manifiesta, tomó el nombre de disfunción cerebral mínima» ${ }^{14}$. Finalmente, se ha ido profundizando más en dicha perturbación hasta llegar a lo que se conoce actualmente como Trastorno del Déficit de Atención e Hiperactividad.

No obstante, a la hora de establecer el factor determinante que decreta qué elementos son los verdaderamente causantes de esta perturbación, cabe destacar la investigación desarrollada por Rose, donde extrapola que, actualmente, se especula que la causa principal para entender el surgimiento del TDAH se encuentra, equivocadamente, dentro de la cabeza del niño. Así, este mismo autor determina que los verdaderos síntomas se entremezclan entre una predisposición genética y unos factores de riesgo sociales, los cuales podrían tener su inicio en la inestabilidad familiar ${ }^{15}$.

En este sentido, Timmi, Taylor, Cannon, McKenzie y Sims establecen que «la hiperactividad no debería ser entendida como una construcción social ni como una enfermedad genética». Así, estos investigadores consideran que los factores sociales probablemente pueden influir en el mayor o menor grado de hiperactividad del individuo ${ }^{16}$.

A raíz de una serie de investigaciones, López Soler, Castro Sáez, Belchí y Romero Medina consideran que el desarrollo del TDAH se configura en torno a tres factores fundamentales, los cuales, a pesar de los repetidos cambios que se han ido produciendo en la

12 CHESS, S., «Diagnosis and treatment of the hyperactive child», en New York State Journal of Medicine, Vol. 60, 1960, págs. 2379-2385.

13 AMERICAN PSYCHIATRIC ASSOCIATION (APA), Diagnostic Statistical Manual of Mental Disorders, Second Edition (DSM-II), Washington D.C., 1968, pág. 50.

14 ODETTE, E., «Potenziamento umano e dirieto penale (*) Il «caso» dell'enhancement cognitivo», en Rivista italiana di diritto e procedura penale, Vol. 55(3), 2012, págs. 975-1015.

15 ROSE, S., Il cervello del ventunesimo secolo. Spiegare, curare e manipolare la mente, Roma, 2007, págs. 321-324.

16 TIMMI, S., TAYLOR, E., CANNON, M., McKENZIE, K., and SIMS, A., «ADHD is best understood as a cultural construct», en The British Journal of Psychiatry, Vol. 184(1), 2004, págs. 8 y 9. 
definición de dicho trastorno, perduran en el pensamiento doctrinal. Así, dichos síntomas son: la desatención, la hiperactividad y la impulsividad ${ }^{17}$.

Por otro lado, recientemente, Reale, Costantino, Sequi y Bonati han realizado un estudio en la Región de Lombardía (Italia) sobre la prevalencia de esta perturbación en adultos que presentaban dicho trastorno durante la edad infantil. Así, en dicha investigación, los autores determinaron que resulta necesario que se lleve a cabo un control más exhaustivo en aquellos sujetos que presentan síntomas de dicha perturbación en la infancia, ya que podría ayudar a su posterior control en la edad adulta ${ }^{18}$.

En este sentido, Hall, Newell Taylor, Sayal, Swift y Hollis han desarrollado unas investigaciones sobre la posible transmisión de este trastorno desde la infancia hasta la adultez, concluyendo que resulta primordial establecer protocolos de actuación primarios para dichos supuestos, ya que existe la posibilidad de que los profesionales encargados de atender a estas personas «puedan estar mal preparados para evaluar y manejar a estos pacientes $\gg{ }^{19}$.

Ante la dificultad de poder clasificar a estas personas, recientemente, Reale y Bonati han llevado a cabo una investigación en 9 de las 20 regiones que conforman la geografía italiana en el que han trabajado la prevalencia del TDAH sobre una muestra de 67.838 sujetos con edades comprendidas entre los 5 y los 17 años. En dicho estudio, estos investigadores aseveran que resulta preciso contar con una base de datos a nivel internacional que facilite la posible evaluación de la prevalencia de las personas que padecen este trastorno ${ }^{20}$.

Como se puede apreciar, establecer una posible relación entre la delincuencia y el trastorno del déficit de atención e hiperactividad en Italia resulta sustancialmente complejo, ya que gran parte de la doctrina italiana centra sus esfuerzos en estudiar la posible correspondencia de esta perturbación con la edad infantil, dejando de lado los casos ocurridos en la edad adulta, por lo que la gran mayoría de sentencias analizadas se centran en las alteraciones que presentan las víctimas, las cuales suelen ser menores de edad.

17 LÓPEZ SOLER, C., CASTRO SÁEZ, M., BELCHÍ, A.I., y ROMERO MEDINA, A., «Descripción clínica: el trastorno por déficit de atención e hiperactividad y los trastornos del comportamiento», en López Soler, C., y Romero Medina, A., TDAH y trastornos del comportamiento en la infancia y la adolescencia, Madrid, 2013, pág. 21.

18 REALE, L., COSTANTINO, M.A., SEQUI, M., and BONATI, M., "Transition to adult mental health services for young people with ADHD», en Journal of Attention Disorders, Vol. 22(6), 2018, págs. 601-608.

19 HALL, C.L., NEWELL, K., TAYLOR, J., SAYAL, K., SWIFT, K.D., and HOLLIS, C., “"Mind the gap" - Mapping services for young people with ADHD transitioning from child to adult mental health services», en BMC Psychiatry, Vol. 13(186), 2013, págs. 1-8.

20 REALE, L., and BONATI, M., «ADHD prevalence estimates in Italian children and adolescents: a methodological issue», en Italian Journal of Pediatrics, Vol. 44(1), 2018, págs. 1-9. 


\section{II.- ESTUDIO DE CASOS}

Para realizar un análisis pormenorizado del TDAH dentro de la jurisprudencia penal italiana, considero esencial proceder al estudio exhaustivo de quince sentencias ${ }^{21}$ dictadas por el Tribunal de Casación Penal de dicho país, donde aparecen reflejadas determinadas particularidades propias de este trastorno, con independencia de ciertos factores identificativos, los cuales han sido un objeto primordial a la hora de llevar a cabo esta investigación.

\section{Situación geográfica}

Para poder relacionar la incidencia del TDAH con determinados censos poblacionales, en primer lugar, considero necesario llevar a cabo un análisis de dichas sentencias para poder esclarecer cuales son las provincias y ciudades italianas donde mayor incidencia exterioriza las acciones delictivas relacionadas con este tipo de trastorno.

En este sentido, los datos relacionados con las tasas de población en Italia han sido recogidos del Istituto Nazionale di Statistica ${ }^{22}$, los cuales serán comparados de forma exhaustiva con la información obtenida de las diferentes sentencias analizadas. Así, en primer lugar, como se puede apreciar en el siguiente gráfico, se procede a clasificar a los habitantes por las diferentes áreas geográficas.

21 Sentenze Cassazione Penale, sezione VI., n. 13.897, [11/02/2010]; Sentenze Cassazione Penale, sezione III., n. 41.702, [13/06/2012]; Sentenze Cassazione Penale, sezione III., n. 28.229, [18/04/2013]; Sentenze Cassazione Penale, sezione III., n. 19.424, [07/02/2014]; Sentenze Cassazione Penale, sezione III., n. 20.419, [07/02/2014]; Sentenze Cassazione Penale, sezione V., n. 36.216, [08/04/2015]; Sentenze Cassazione Penale, sezione V., n. 46.450, [06/05/2015]; Sentenze Cassazione Penale, sezione III., n. 49.165, [06/10/2015]; Sentenze Cassazione Penale, sezione III., n. 50.752, [14/09/2016]; Sentenze Cassazione Penale, sezione VI., n. 41.128, [27/09/2016]; Sentenze Cassazione Penale, sezione IV., n. 43.864, [06/10/2016]; Sentenze Cassazione Penale, sezione VI., n. 10.906, [15/02/2017]; Sentenze Cassazione Penale, sezione I., n. 23.385, [28/04/2017]; Sentenze Cassazione Penale, sezione VI., n. 38.993, [06/06/2017]; Sentenze Cassazione Penale, sezione VI., n. 25.248, [03/05/2018].

22 Recurso electrónico disponible en: http://demo.istat.it/pop2018/index_e.html. 


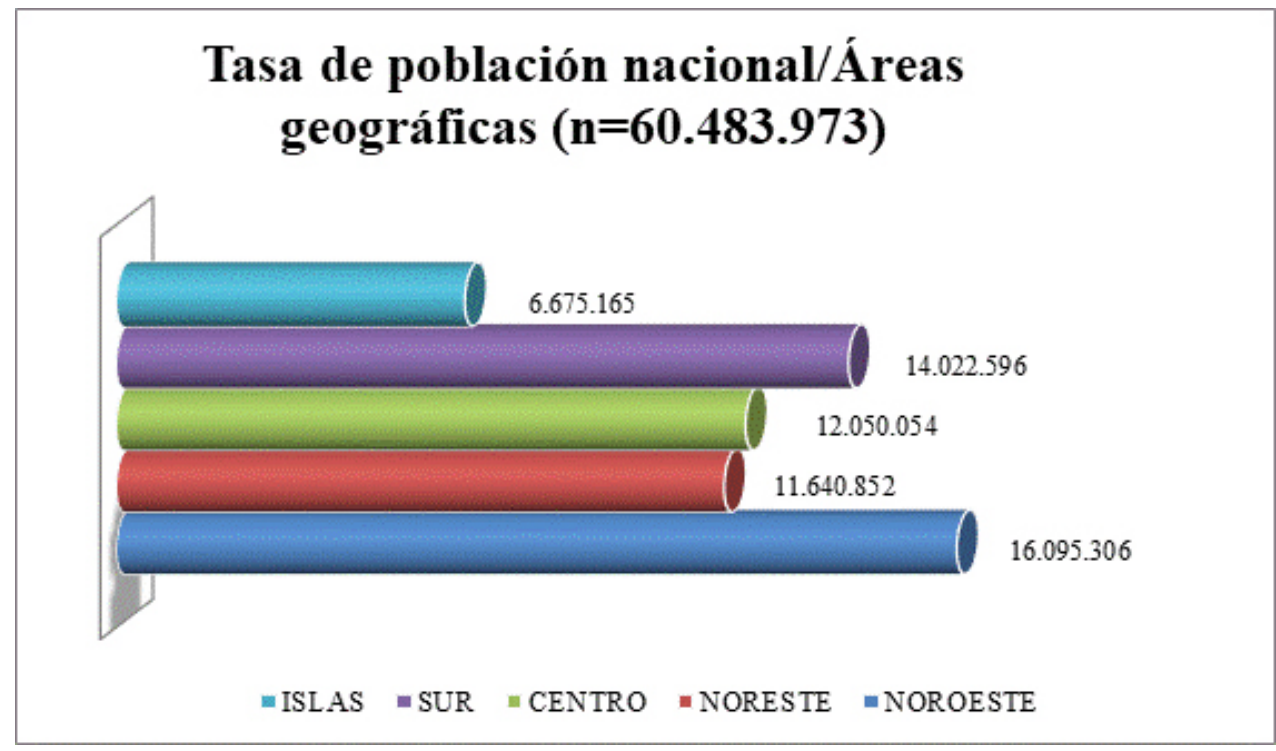

Como se puede observar en el gráfico anterior, a fecha de 1 de enero de 2018, el área territorial que presenta una mayor tasa de ciudadanos es la representada por la parte noroeste del país, seguida por el segmento poblacional ubicado en la parte sur. Por contra, según los datos oficiales, la zona donde se aprecian unos porcentajes más bajos es la que hace referencia a las islas del país transalpino.

En este sentido, como se puede apreciar en el siguiente gráfico, una vez analizadas las sentencias señaladas, existe gran semejanza entre las diferentes áreas geográficas que dividen Italia. De este modo, se observa como los porcentajes muestran cómo serán las zonas Central y Noreste las que presentan unos índices un poco más elevados. Por último, llama la atención que no se haya podido obtener ningún suceso ocurrido en las Islas.

\section{Áreas geográficas relacionadas con sucesos de TDAH $(\mathbf{n}=15)$}

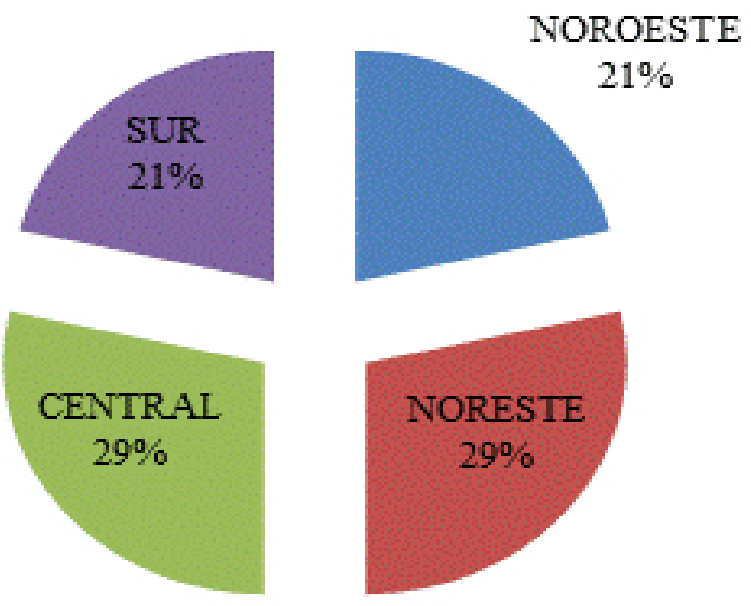


No obstante, debido a la gran densidad del país, considero necesario pasar al análisis de cada una de las áreas geográficas de forma independiente, ya que así se podrá observar en que zona concreta se produce un mayor número de casos relacionados con este tipo de trastorno.

En primer lugar, como se puede apreciar en el siguiente gráfico, en la zona del Noroeste del país se producen tres sucesos relacionados con el TDAH, de los cuales dos tienen lugar en Milán, mientras que otro en Brescia. En este sentido, se observa como de las cuatro áreas en las que se divide dicha zona, sólo una tiene incidencia en la proyección de este trastorno.

\section{Sucesos de TDAH en el noroeste $(n=3)$}

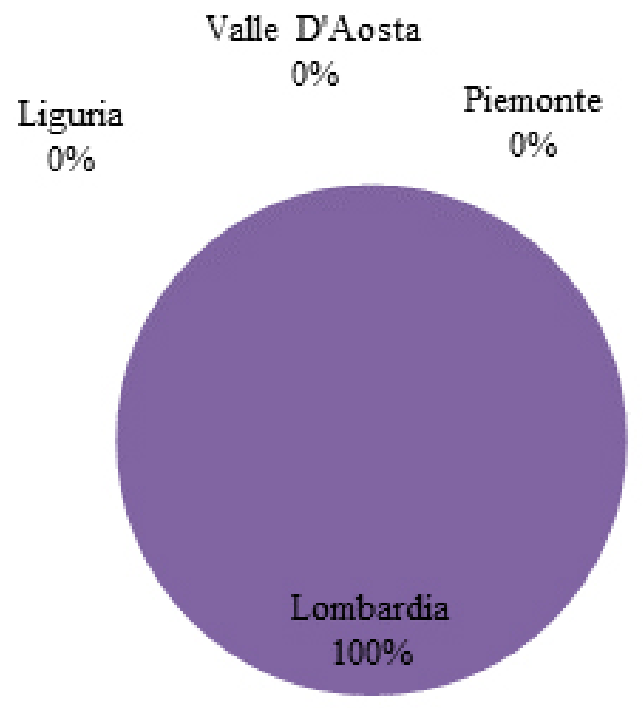

Seguidamente, la zona correspondiente al noreste del país exterioriza un mayor número de acontecimientos, dividiéndose en dos regiones distintas, tanto en Trentino como en Emilia-Romagna, donde se puede apreciar como existe un porcentaje mucho más elevado. 


\section{Sucesos de TDAH en el noreste $(n=4)$}

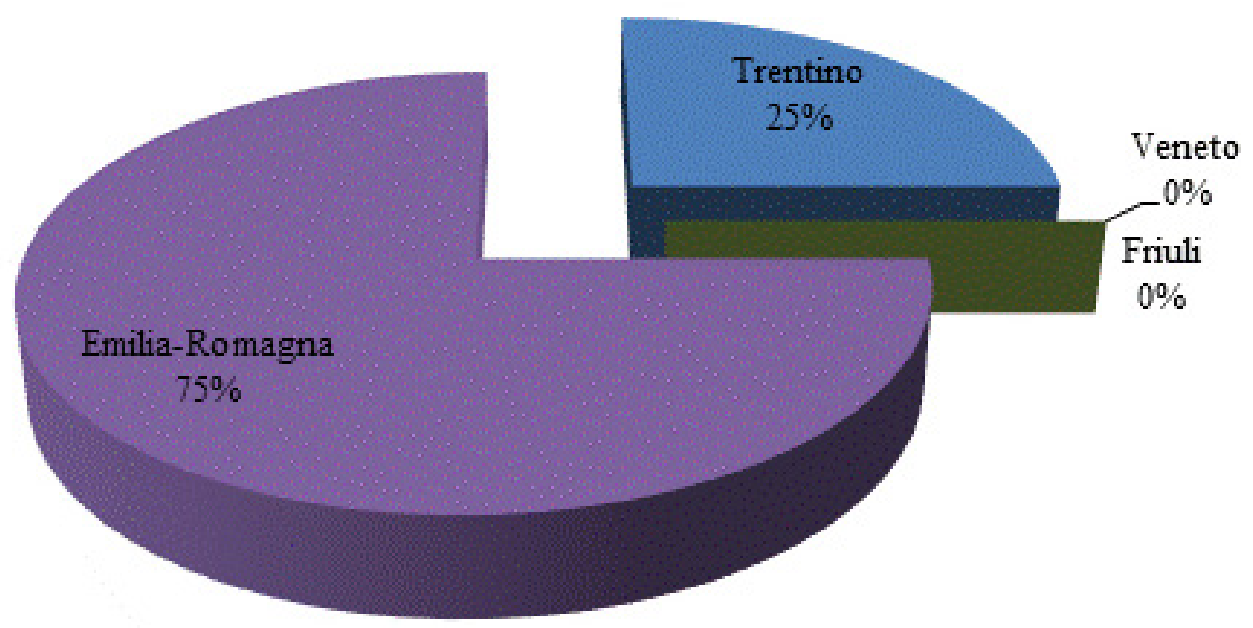

En lo que respecta al área centro, los datos analizados muestran la presencia de un mayor reparto de sucesos relacionados con el TDAH entre las distintas regiones que conforman la totalidad de dicho espacio geográfico. Así, tras el estudio de las diferentes sentencias, Lacio se configura como la zona donde se origina un mayor índice de acontecimientos en los que tiene lugar este trastorno.

\section{Sucesos de TDAH en la zona centro $(n=4)$}

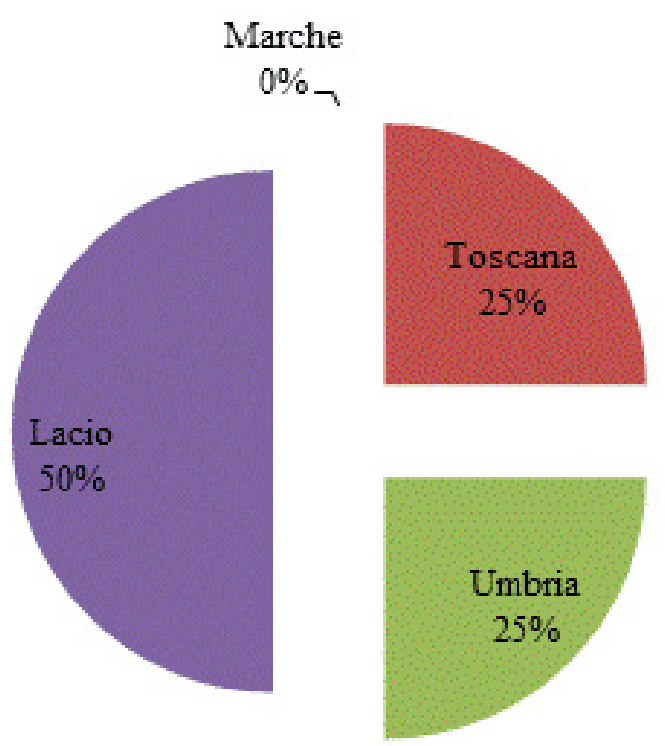


Por último, la zona sur, a pesar de ser el área geográfica donde se ubica un mayor número de regiones, muestra cierta similitud con el noroeste del país, ya que todos los acontecimientos tienen lugar en una sola circunscripción, no existiendo hasta la fecha ningún suceso relacionado con el TDAH en el resto de demarcaciones.

\section{Sucesos de TDAH en la zona sur $(n=3)$}

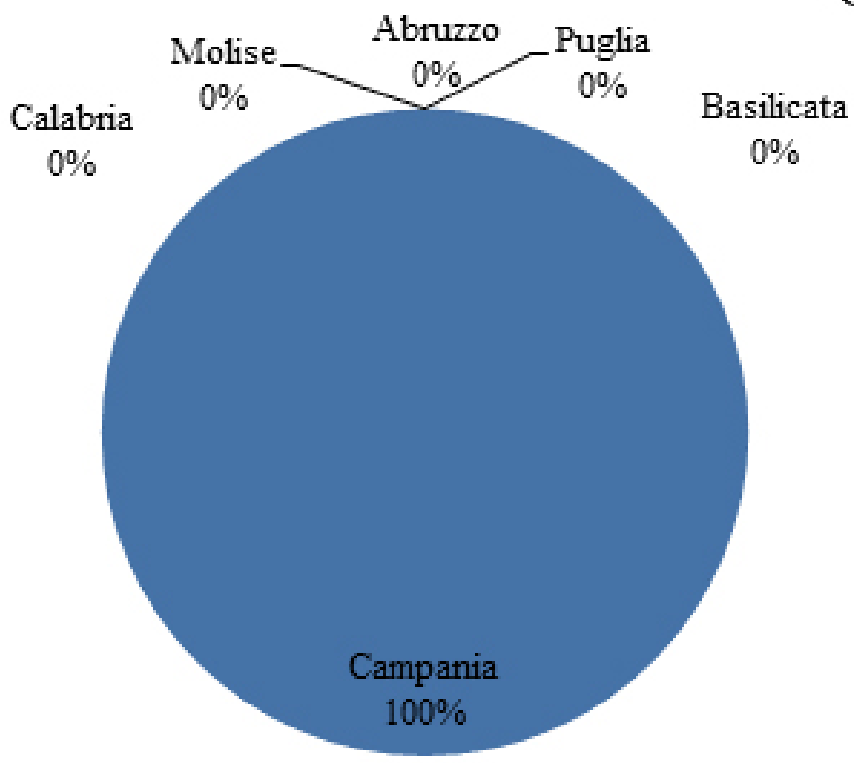

Así, una vez desglosada la distribución de sucesos relacionados con el TDAH dentro de cada una de las diferentes áreas geográficas en las que se distribuye Italia, considero necesario pasar a identificar cuáles son las ciudades donde existe un mayor porcentaje acontecimientos donde el agresor o la víctima presenta síntomas propios de este trastorno.

En este sentido, en el siguiente gráfico se observa como los mayores porcentajes se reflejan en las ciudades de Bolonia y Nápoles, produciéndose en cada una de ellas tres situaciones en las que alguno de los sujetos exterioriza este trastorno, representando estas dos poblaciones un $44 \%$ del total de los sucesos analizados. En el lado opuesto se encontrarían las ciudades de Brescia, Trento, Florencia y Perugia, con tan solo una sentencia relacionada con el TDAH. 


\section{Porcentaje del TDAH en diferentes ciudades $(n=15)$}

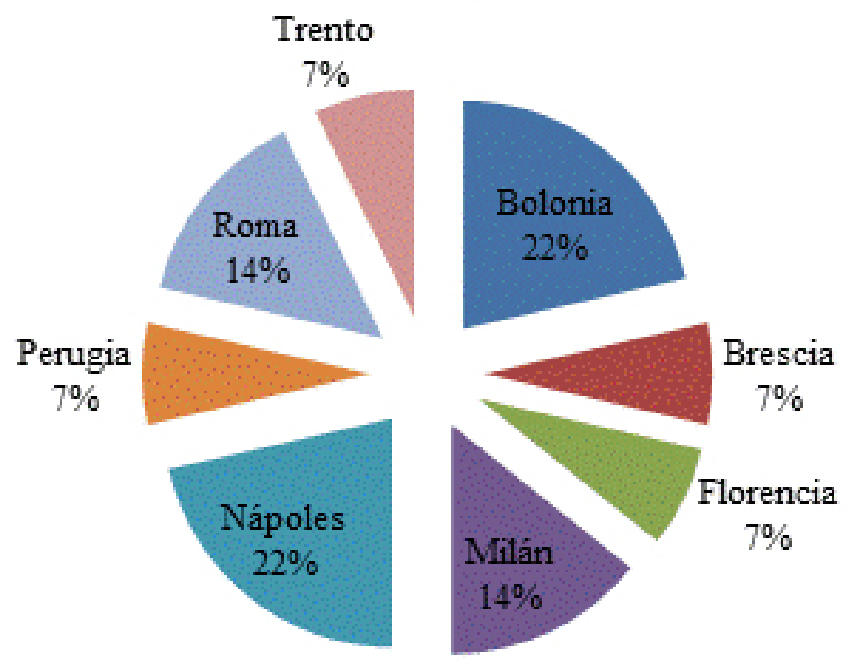

En referencia al gráfico anterior, considero imprescindible mencionar que las ciudades señaladas se distribuyen en las diferentes áreas geográficas de la siguiente forma:

F. Milán y Brescia pertenecen a Lombardía, la cual está situada en la zona del noroeste.

G. Bolonia se encuentra dentro de Emilia-Romagna; mientras que Trento está situada en Trentino, ambas pertenecientes a la zona del noreste.

H. Respecto a la zona central, cabe mencionar que Roma está ubicada en Lacio; Florencia en la Toscana y Perugia en Umbría.

I. Finalmente, Nápoles se halla en la Campania; más concretamente, en el área sur.

Teniendo en consideración lo descrito anteriormente, se extrapola que las ciudades que presentan un mayor índice de casos relacionados con el TDAH pertenecen al área sur y a la zona del noreste. En este sentido, teniendo presentes los datos concernientes a las tasas de población nacional, se puede apreciar como dichas áreas representan la segunda y la cuarta zona en volumen de habitantes.

\section{Circunstancias personales}

\subsection{Edad de la pareja penal}

Al hablar sobre la edad de las personas que han participado de forma activa o pasiva en la comisión de un acto delictivo, hemos de tener en cuenta que dicho parámetro 
se configura como un elemento esencial a la hora de proceder al estudio de las diferentes variables que pueden incidir en la ejecución de una determinada infracción. Así, Morillas Fernández anticipa que el análisis y estudio de dicha variable se ha convertido en una constante premisa por parte de la doctrina criminológica ${ }^{23}$.

En lo que respecta a la edad de las personas que padecen el TDAH, con independencia de si han sido víctimas o agresores, el estudio de las sentencias anteriormente citadas muestra como existe una leve mayoría de menores de edad que sufren dicho trastorno.

\section{Franjas de edad $(n=15)$}
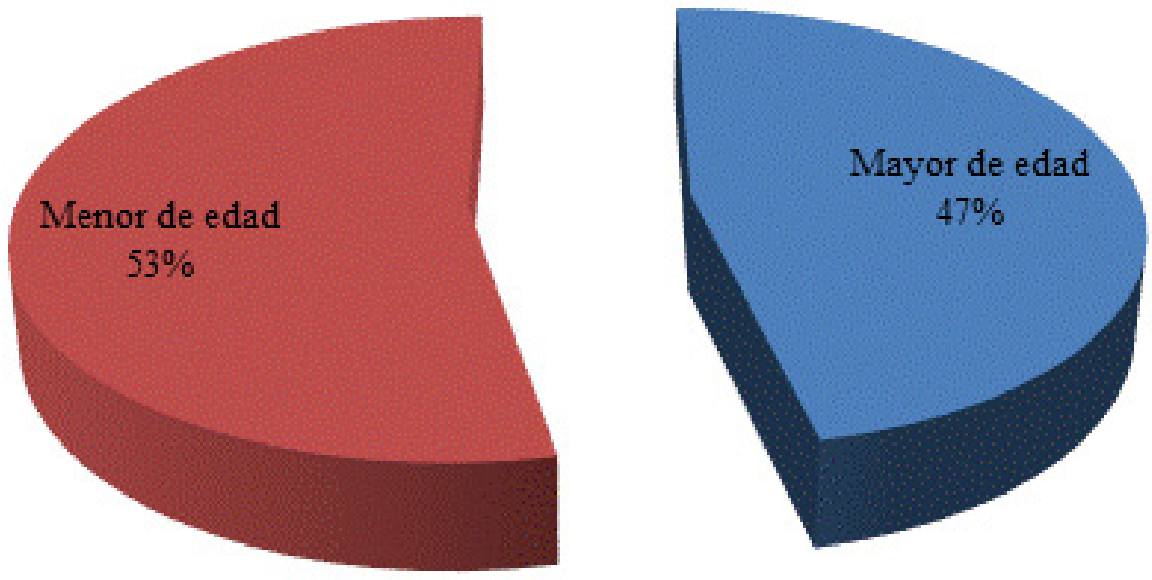

En este sentido, en relación con un estudio llevado a cabo en España sobre 40 sentencias condenatorias, los resultados son totalmente opuestos a los observados en las resoluciones analizadas en el país italiano, ya que muestran cómo serán los mayores de edad los que presentarán unos porcentajes de padecimiento de TDAH mucho más elevados que los menores ${ }^{24}$.

Las tasas de delincuencia relacionadas con la mayoría o menoría de edad en Italia muestran como existe una amplia diferencia entre unos y otros. Así, en el siguiente gráfico se puede observar como en el año 2016 las cifras exteriorizan que los adultos; es decir,

23 MORILLAS FERNÁNDEZ, D.L., «Análisis de las principales variables de la delincuencia juvenil en España», en Revista de Derecho, Empresa y Sociedad, REDS, Vol. 3, Época I, 2013, pág. 198.

24 PINTADO ALCÁZAR, A., «Análisis criminológico de la relación TDAH/delincuencia en España», en Peris Riera, J., El trastorno por déficit de atención e hiperactividad y su repercusión en la responsabilidad penal, Madrid, 2017, págs. 157-184. 
aquellos cuya edad supera los 18 años, presentan unos datos porcentuales muy elevados respecto a los mostrados por los menores ${ }^{25}$.

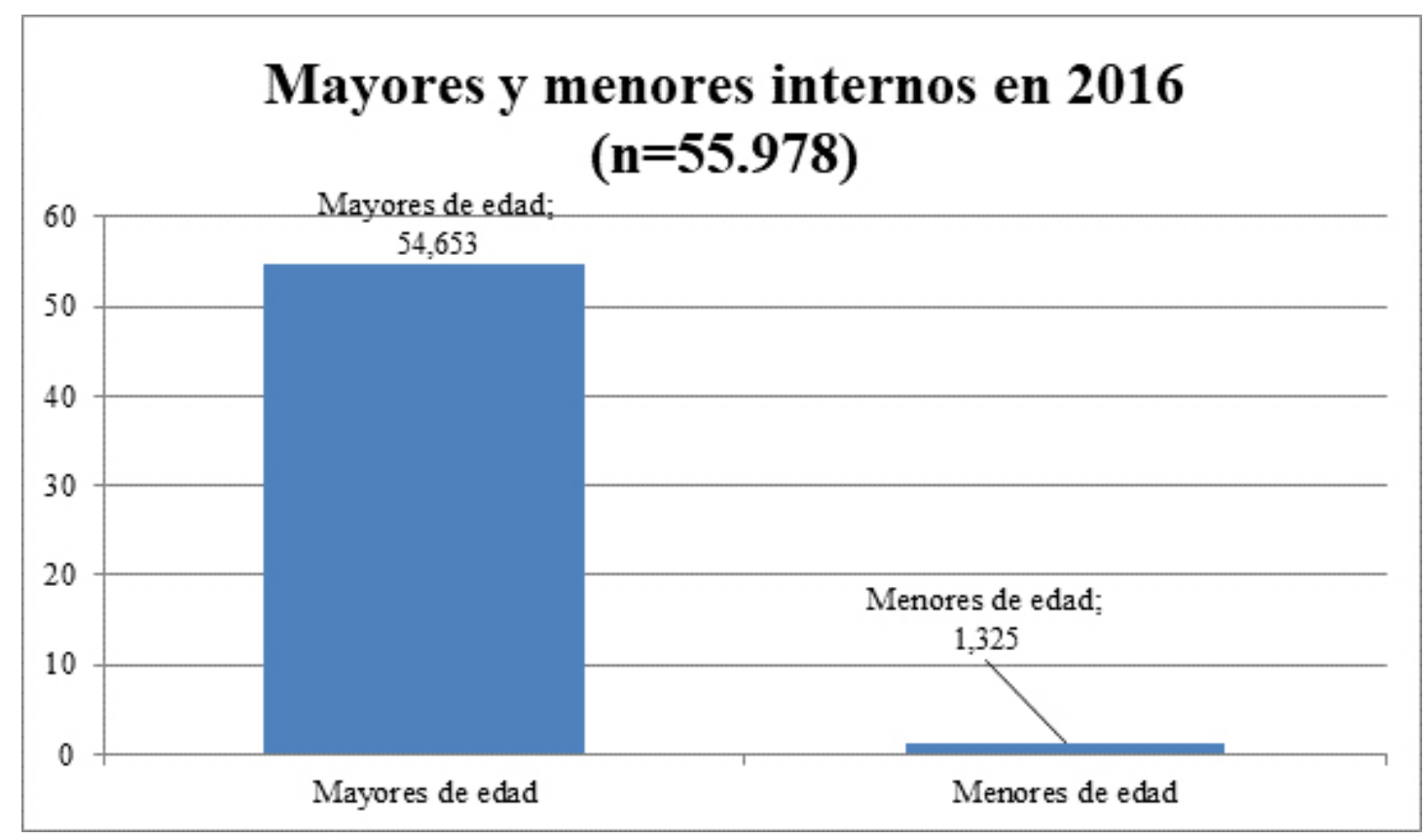

En este sentido, en un intento de pasar a desglosar e individualizar a cada uno de los diversos grupos de edad, considero fundamental observar la evolución de la delincuencia desde el año 2008 hasta el 2016, siendo éste el último período temporal en el que se han encontrado datos oficiales.

Respecto a los internos menores de edad, el siguiente gráfico muestra como durante los primeros años se produjo un incremento del número de sujetos con edades inferiores a los 18 años que estaban recluidos en centros penitenciarios especiales, adaptados a sus correspondientes edades.

Así, dicha evolución llegó a su punto más elevado en el año 2011, cuando el total de menores internos estaba cifrado en 1428 menores de edad. Posteriormente, durante los tres años siguientes, se produjo un descenso del número de personas recluidas en estos centros, volviendo a aumentar gradualmente hasta la fecha en la que se han obtenido datos oficiales; es decir, en 2016.

25 Recurso electrónico disponible en: http://appsso.eurostat.ec.europa.eu/nui/submitViewTableAction.do 


\section{Menores de edad $(n=11.500)$}

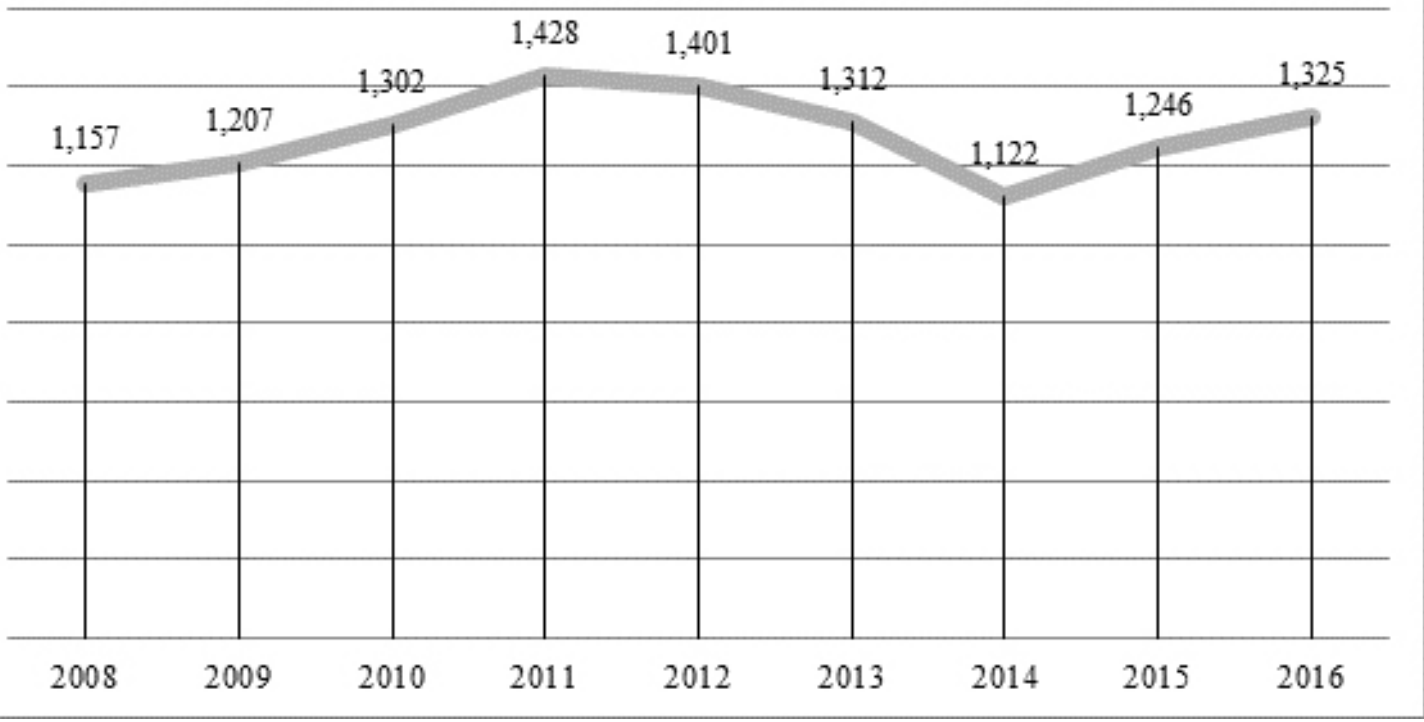

Por otro lado, en lo que respecta a las personas reclusas en centros penitenciarios italianos, los datos analizados muestran un elevado incremento durante los primeros años del citado estudio, ya que desde el año 2008 hasta el 2010 se produjo una cifra de ingresos cercana a los 10000 internos, llegando al cómputo total de 67961 internos en las diferentes prisiones del país transalpino.

En referencia a la cifra antes señalada, es a partir del 2010 cuando se produce un descenso anual del número de internos en prisiones italianas, llegando a ser el año 2015 el que exterioriza unos porcentajes más reducidos respecto a los acontecidos en los períodos propios del estudio.

\section{Mayores de edad $(n=546.453)$}

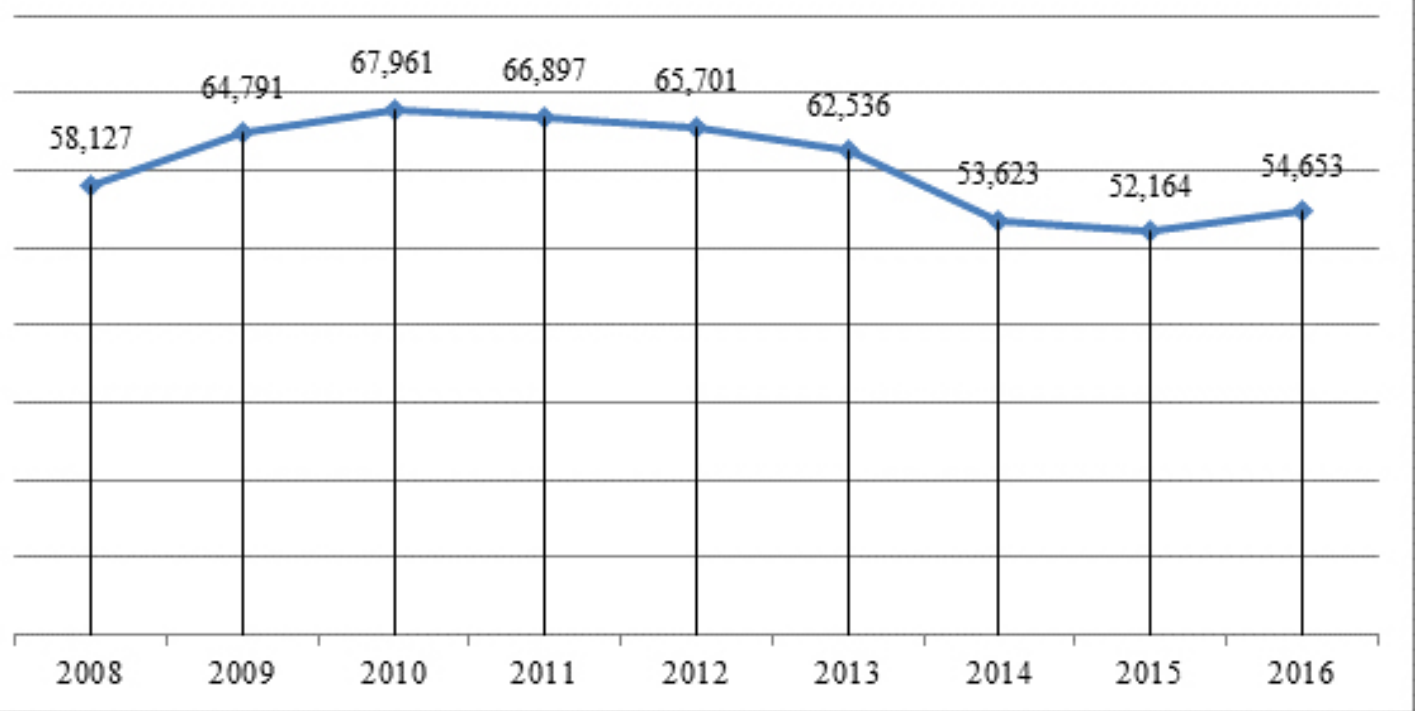




\subsection{Sexo de los implicados}

En lo que respecta al sexo de las personas que padecen este trastorno en Italia, sin prestar atención a si se configuran como delincuentes o víctimas del hecho delictivo, las sentencias analizadas muestran como los hombres presentan un índice mucho más elevado al que exteriorizan las mujeres.

En este sentido, Colombo ha realizado una investigación centrada en las características principales que se relacionan con determinados crímenes cometidos en el país transalpino. Así, los resultados de su estudio muestran como los hombres se configuran como sujetos más propensos a llevar a cabo una acción delictiva; por otro lado, las mujeres suelen presentar índices más elevados en lo concerniente al sujeto pasivo del crimen $^{26}$.

\section{Sexo de las personas relacionadas con el TDAH $(n=19)$}

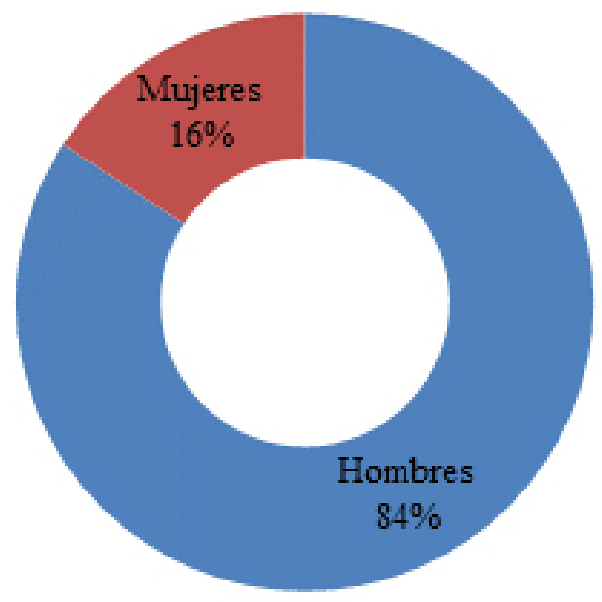

Cianciola afirma que los hombres siempre han sido más vehementes que las mujeres, por lo que se encuadran como sujetos más propensos para llevar a cabo la comisión de determinados actos violentos. Así, a pesar de la amplia diferencia porcentual existente entre ambos sexos, esta investigadora afirma que las mujeres han ido incrementado las tasas delictivas y determina que «en los últimos años ha habido un ligero aumento de este fenómeno, solo en lo que respecta a algunas categorías de delitos, entre los que no se encuentran los cometidos contra las personas». Finalmente, la autora afirma que las personas del género femenino se siguen configurando como los principales sujetos pasivos del delito ${ }^{27}$.

26 COLOMBO, A., «Gli omicidi Italia. Tendenze e caratteristiche dall'Unità a oggi», en Rassegna Italiana di Criminologia, Vol. 4, 2011, págs. 52-64.

27 CIANCIOLA, G., Genere e crimine nella società postmoderna. La donna Kamikaze, Roma, 2009, pág. 15. 
Como se puede apreciar en el siguiente gráfico, las sentencias analizadas exteriorizan la existencia de un mayor índice de sujetos pasivos que padecen este tipo de trastorno. En este sentido, Aguilar Cárceles se plantea la posibilidad de que aquellas personas que tengan TDAH podrán tener una cierta predisposición a convertirse en sujetos especialmente vulnerables, al igual que sucede con otras alteraciones previstas en los diferentes manuales específicos de dicha materia ${ }^{28}$.

\section{Sujetos con TDAH $(n=15)$}
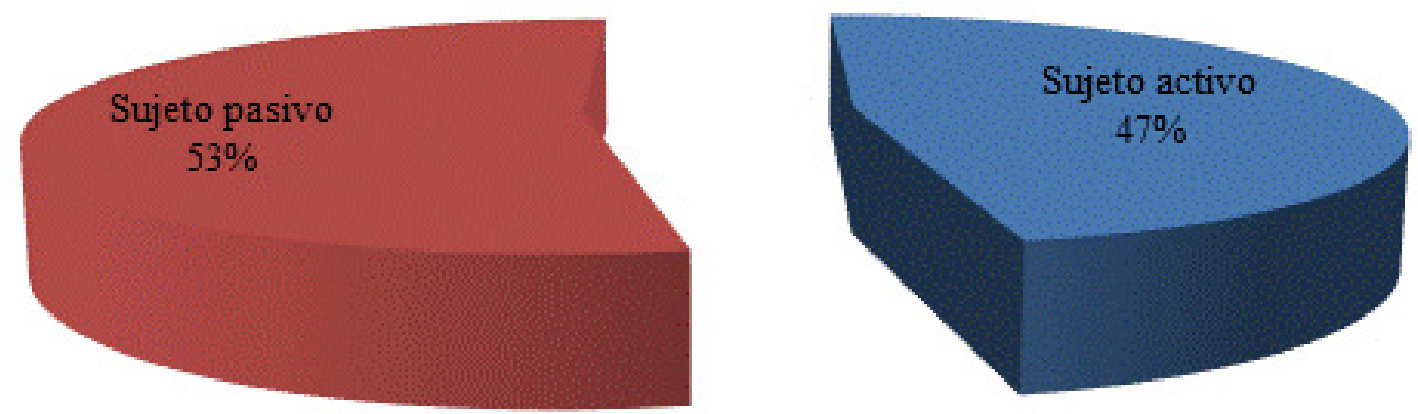

\section{Características penales}

\subsection{Delitos cometidos}

En referencia a los crímenes cometidos por aquellas personas que fueron enjuiciadas en las sentencias analizadas, en el siguiente gráfico se puede observar como existe una disparidad de actos delictivos. Así, se aprecia cómo serán las infracciones realizadas dentro del ámbito familiar las que llevarán aparejadas unas mayores tasas porcentuales. En el lado opuesto se encuentra el homicidio, el tráfico de drogas y la falsedad documental, presentando cada una de ellas unos porcentajes muy escasos en relación al resto de actos cometidos.

En este sentido, haciendo referencia al delito referido al maltrato familiar, el Código Penal Italiano tiene entre sus epígrafes un título destinado específicamente a los delitos contra la familia. Así, la Corte de Casazzione Penale engloba dentro de la palabra «maltrato» todas aquellas acciones que tienen su punto de partida en amenazas, injurias, humillaciones o sufrimientos morales, entre otros ${ }^{29}$.

28 AGUILAR CÁCERLES, M., El trastorno por déficit de atención e hiperactividad (TDAH). Aspectos jurídico-penales, psicológicos y criminológicos, Madrid, 2014, pág. 391.

29 Sentenze Cassazione Penale, sezione III., n. 4.905, [17/05/1985]. 
No obstante, Trinci y Farini, al hablar del delito de maltrato familiar, establecen que para que se produzca es necesario que el sujeto activo esté vinculado al pasivo por una relación familiar, una relación de autoridad o educativa, siempre que así venga reflejado de forma específica en la legislación correspondiente ${ }^{30}$.

\section{Diferentes crímenes cometidos $(n=14)$}

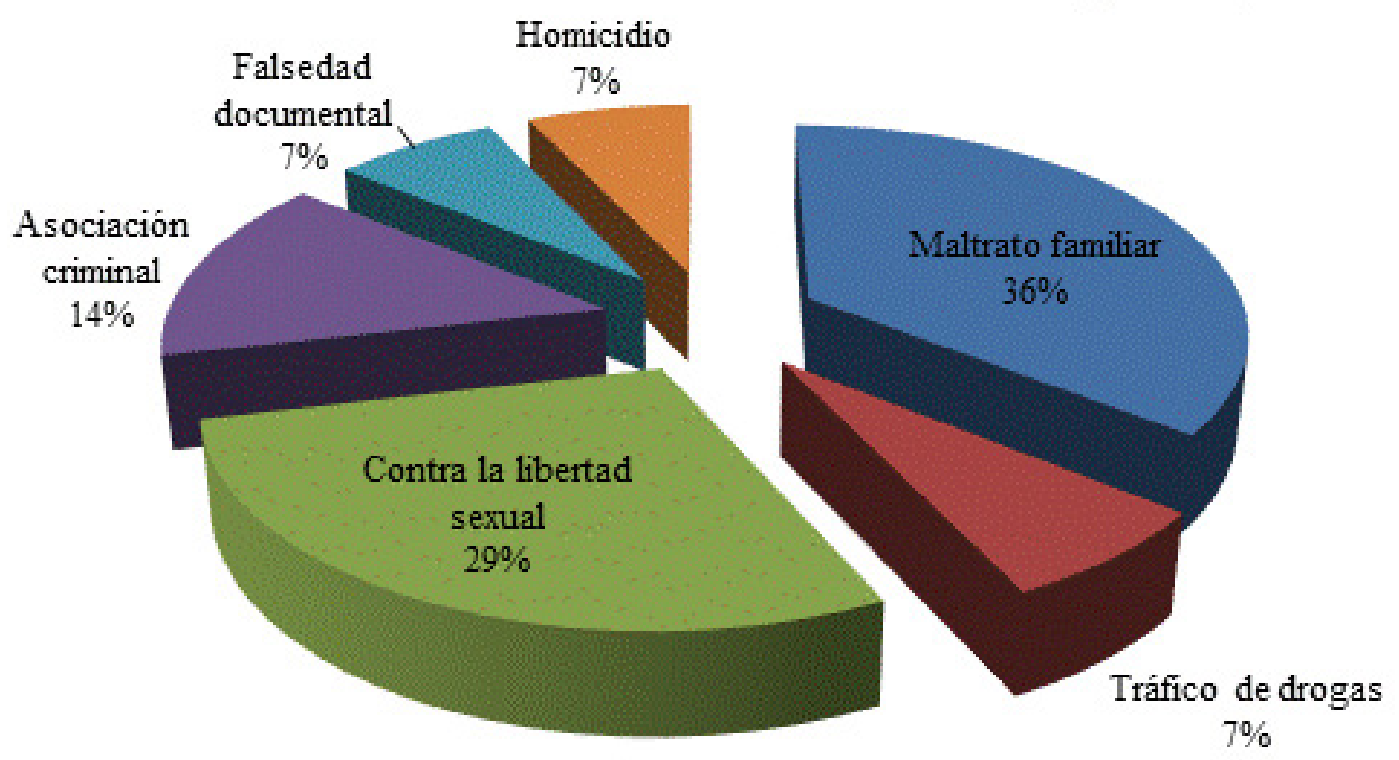

Por otro lado, el gráfico anterior muestra cómo serán los delitos cometidos contra la libertad sexual los que se configuran como uno de los crímenes cometidos en Italia que mas relación tienen con el padecimiento del TDAH por parte de cualquiera de los miembros de la pareja penal. En este sentido, uno de los principales cimientos en los que se mueve la última modificación del Código Penal italiano tiene su fundamento en el intento de poder proteger aún más a la víctima, endureciendo las penas correspondientes a este tipo de delitos ${ }^{31}$.

En referencia a los delitos contra la libertad sexual, Delpino y Pezzano afirman que «es posible distinguir dos tipos de acciones encuadradas dentro de la violencia sexual: la llevada a cabo por la acción directa en la persona lesionada; y la violencia sexual cometida por inducción $»^{32}$.

Posteriormente, como se puede apreciar en dicho gráfico, los delitos de asociación criminal también presentan unos porcentajes relativamente elevados dentro del cómputo total de acciones delictivas relacionadas con este tipo de trastorno. En este sentido, en referencia a este tipo de delitos, Borrelli, al hacer un análisis exhaustivo sobre dicho delito

30 TRINCI, A., y FARINI, S., Compendio di Diritto Penale. Parte Speciale, Roma, 2018, pág. 521.

31 ROMANO, B., FINAZZO, S., MANNO, M.A., y MINO, A., «Reati contro la persona», en Grosso, C.F., Padovani, T., y Pagliaro, A (Dirs.)., Trattato di diritto penale, Vol. XIV., Milán, 2016, pág. 239.

32 DELPINO, L., y PEZZANO, R., Manuale di Diritto Penale. Parte Speciale, Nápoles, 2018, pág. 533. 
en la jurisprudencia italiana, el cual se encuentra regulado en el artículo 416 del Código Penal de dicho país, afirma que «en la doctrina se consideró en una clave socio-criminológica más que penalista» ${ }^{33}$.

Finalmente, se puede observar como los delitos de tráfico de drogas, falsedad documental y el homicidio son los que tienen una menor representatividad porcentual dentro del conglomerado de acciones delictivas que presentan una relación directa con la presencia del TDAH en cualquiera de los miembros de la pareja penal.

En relación con la afirmación anteriormente aportada, en lo relativo a los delitos cometidos contra la falsedad documental, De Amicis afirma que para hablar sobre falsificación hay que tener en cuenta que «se crea un documento cuyo autor aparente no es el real; es decir, cuando las alteraciones del documento se modifican para modificar su esencia material; la falta de veracidad, por otro lado, ocurre cuando el documento, incluso si proviene del sujeto que parece ser el autor, contiene declaraciones falsas» ${ }^{34}$.

Por otro lado, al hacer referencia al delito de homicidio, Palermo y Mastronardi afirman que dicho crimen se configura como un fenómeno complejo, el cual puede ser provocado por diversas causas. En este mismo sentido, dichos autores establecen que «para entender su génesis, han sido formuladas numerosas teorías explicativas, pero ninguna de ellas parece capaz de proporcionar, por si misma, una explicación exhaustiva de la violencia homicida ${ }^{35}$.

\subsection{Circunstancias modificativas de la responsabilidad penal}

Este tipo de contextos penales se fundamentan, principalmente, en las circunstancias agravantes y/o atenuantes. Su función básica viene referida en un intento por aumentar o reducir la condena impuesta en base a una serie de connotaciones punitivas que el legislador ha considerado necesarias.

En referencia a las circunstancias agravantes comunes reguladas en el Código Penal italiano, el artículo 61 de dicha compilación viene a establecer que para que se produzca un posible aumento de la pena impuesta, debe existir alguna de las siguientes situaciones penales:

1. Haber actuado por razones abyectas o inútiles

33 BORRELli, G., «Dei delitti contro l'ordine pubblico», en Lattanzi, G., y Lupo, E., Codice Penale. Rassegna di giurisprudenza e di dottrina, Vol. IX, Milán, 2015, pág. 137.

34 DE AMICIS, G., «Dei delitti contro la fede pubblica», en Lattanzi, G., y Lupo, E., Codice Penale ... Vol. VI, cit., págs. 212-213.

35 PALERMO, G.B., y MASTRONARDI, V., «L'omicidio. Profili comparatistici Italia-USA», en Supplemento alla Rivista di Psichiatria, Vol. 47(4), 2012, págs. 1-10. 
2. Haber cometido el delito para ejecutar u ocultar otro, para lograr o asegurar, para ellos o para otros, el producto, la ganancia o el precio, o la impunidad de otro delito.

3. Haber actuado en los delitos, a pesar de la predicción del evento.

4. Haber utilizado la tortura, o haber actuado con crueldad hacia las personas.

5. Haberse aprovechado de las circunstancias de tiempo, lugar o persona, también con referencia a la edad, para obstaculizar la defensa pública o privada.

6. Que el delincuente cometiera el delito durante el tiempo en el que evadió voluntariamente la ejecución de una orden de arresto o encarcelamiento.

7. En delitos contra la propiedad, causar a la persona ofendida un daño material grave.

8. Haber agravado o intentado agravar las consecuencias del delito cometido.

9. Haber cometido el delito con abuso de poderes, o mediante la violación de los deberes inherentes a una función pública o a un servicio público.

10. Haber cometido el acto contra un funcionario público o una persona de cargo de un servicio público, o vestido como un Ministro de culto católico o de un culto admitido por el Estado, o contra un agente diplomático o consular de un Estado extranjero, en el acto o por el cumplimiento de su función o servicio.

11. Haber cometido el delito con abuso de autoridad o de relaciones domésticas, o con abuso de relaciones de laborales.

11-bis. Haber cometido el hecho mientras el culpable se encuentra ilegalmente en territorio nacional.

11-ter. Haber cometido un delito contra un menor de edad, tanto dentro como cerca de instituciones educativas o de capacitación.

11-quarter. Haber cometido un delito mientras el delincuente fue admitido para serle impuesta una medida alternativa al encarcelamiento en prisión.

11-quinquies. Haber cometido delitos contra la vida y la seguridad individual, contra la libertad personal, o contra el maltrato familiar, en presencia de menores de dieciocho años, o causándole daños a ellos o a personas embarazadas. 
En referencia a los contextos agravantes detallados anteriormente, cabe destacar que, si se observa la concurrencia de alguna de las circunstancias descritas, el condenado podrá ver incrementada potencialmente la pena que se le iba a imponer en un primer momento.

Así, en palabras de Zingirian, «obviamente, para algunos factores agravantes, el criterio aplicativo de la culpa no será suficiente, ya que por su propia formulación implican necesariamente el conocimiento, y la voluntad, de los elementos integradores de la circunstancia misma $»^{36}$.

En el lado opuesto se encuentran las circunstancias atenuantes, las cuales tienen una función encaminada a lograr una disminución de la posible pena a imponer inicialmente, ya que se producen una serie de acciones concretas que han sido consideradas como tal. En este sentido, el artículo 62 del Código Penal italiano afirma que, para ser considerada como una circunstancia atenuante, es necesario que concurra alguna de las citadas acciones:

1. Haber actuado por razones de particular valor moral o social.

2. Haber reaccionado con ira, determinado por un hecho injusto cometido por los demás.

3. Haber actuado por sugerencia de una multitud en agitación, cuando no se trata de reuniones y el culpable no es un delincuente habitual o profesional.

4. Haber cometido delitos contra la propiedad, causando a la persona ofendida un daño pecuniario especial, o, en delitos determinados por motivos de lucro, haber actuado para lograr, o haber obtenido, un beneficio de tenuidad especial, cuando incluso el evento dañino o peligroso es de tenue especialidad.

5. Estar involucrado en la determinación del evento, junto con la acción u omisión del culpable.

6. Antes de la sentencia, reparar completamente el daño causado, compensándolo y, cuando sea posible, mediante reembolsos; o, de manera espontánea y efectiva, intentar mitigar las consecuencias perjudiciales o peligrosas del delito.

En este sentido, y teniendo presentes las diferentes circunstancias modificativas de la responsabilidad penal que vienen reguladas en el Código Penal italiano, en el siguiente gráfico se puede apreciar como existe un porcentaje más elevado de actuaciones atenuan-

36 ZINGIRIAN, G., La danza delle circostanze: Profili applicativi degli accidentalia delicti, Vicalvi, 2015, pág. 29. 
tes relacionadas con la presencia del TDAH en alguno de los miembros que componen la pareja penal de las distintas sentencias analizadas.

\section{Circunstancias modificativas de la responsabilidad penal $(n=8)$}

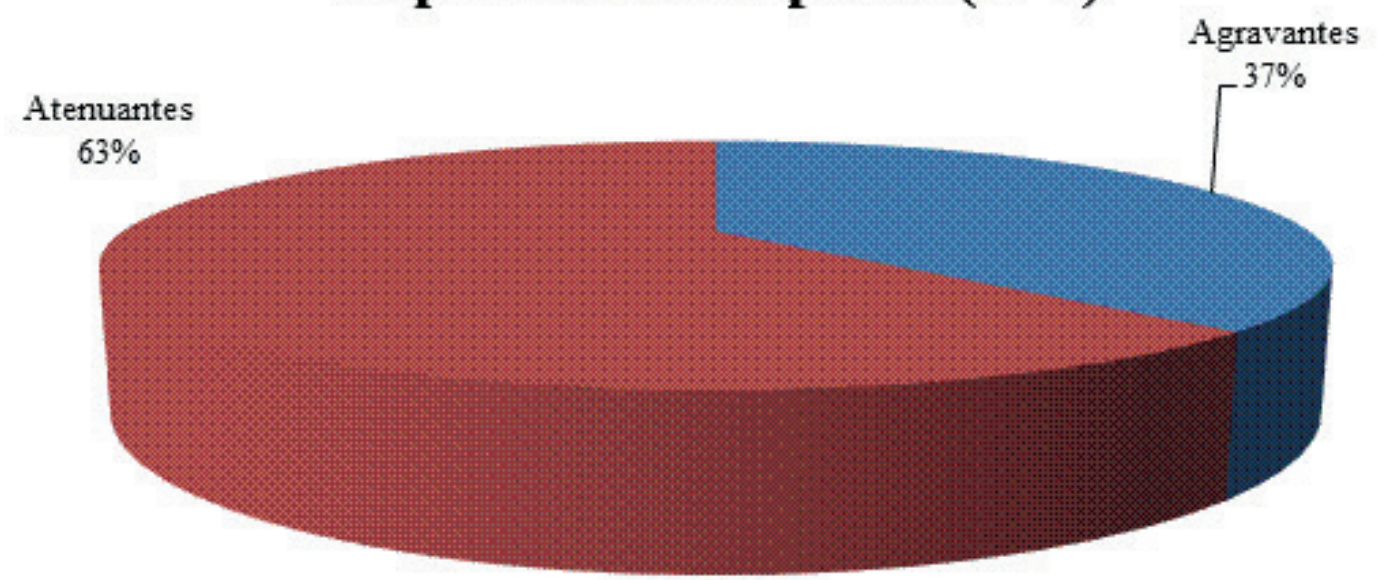

No obstante, también resulta necesario exponer que dichas circunstancias modificativas de la responsabilidad penal pueden repartirse por una gran parte del articulado del Código Penal italiano. Así, por ejemplo, las sentencias analizadas muestran como, para hablar de alguna circunstancia atenuante, no siempre se fundamentan en el artículo 62 de dicho Código.

En este sentido, tras analizar las sentencias condenatorias obtenidas, se puede observar como la distribución de las circunstancias atenuantes y agravantes presentan una gran disparidad. Así, de las cinco situaciones en las que se produce una reducción de la pena, se puede apreciar como en dos de los casos estudiados se procederá a la aplicación del artículo 62 bis, el cual hace referencia a las atenuantes genéricas y en las que se establece la posible intervención del Juez para la aplicación de dichas circunstancias modificativas de la responsabilidad penal en supuestos ajenos a los establecidos para determinar las atenuantes comunes, siempre que este las considere idóneas para justificar la reducción de la condena. Por otro lado, el resto de acciones estarán repartidas en dicho Código Penal y vienen referidas a los siguientes supuestos; a) cometer el hecho encontrándose, por alguna enfermedad concreta, en un estado de ánimo tal que haya provocado una disminución en la capacidad de entender y comprender la acción cometida; b) la realización de un delito de violencia sexual con una menor gravedad de lo previsto inicialmente; c) la exigua colaboración en un delito relacionado con el tráfico de drogas. 
Por otro lado, en lo referente a las circunstancias agravantes, las sentencias analizadas exteriorizan una serie de situaciones concretas; a) la comisión del acto delictivo concurriendo un número elevado de infractores; b) realizar una acción criminal relacionada con el tráfico de drogas concurriendo gran cantidad de estupefacientes; c) haber cometido el delito en el momento en el que el delincuente se encuentra disfrutando de un permiso de salida por la comisión de una acción anterior.

\section{CONCLUSIONES}

Teniendo en cuenta todo lo desarrollado anteriormente, se puede apreciar cómo no existe una alta incidencia del TDAH dentro de la jurisprudencia penal italiana, hecho que puede repercutir directamente en el tipo de sentencia que se le puede imponer a un determinado delincuente. Ante tal problemática, considero fundamental que se realicen más estudios relacionados con este tipo de trastorno desde una perspectiva jurídico-penal y criminológica.

Respecto al modelo italiano, como ya he mencionado, falta regulación específica que se cimente en el estudio relacionado con este tipo de personas. Muestra de ello, se puede apreciar en el número de sentencias que han podido ser analizadas, las cuales, en su totalidad, provienen de la Corte de Casación Penal italiana.

En este sentido, y para centrarme en el objeto final pretendido con la realización de este estudio, el cual se basa en intentar encontrar una interacción o, por el contrario, una desemejanza entre este trastorno y la comisión de algún tipo de acción delictiva, considero que deberían estudiarse de forma minuciosa a todas aquellas personas que se encuentren privados de libertad, hecho que ayudaría a la realización de un perfil más concreto referido a este tipo de sujetos.

Así, considero fundamental proceder al examen y análisis de todas aquellas personas que durante la etapa infantil han sido diagnosticadas con TDAH. En este sentido, resultaría conveniente que dicho estudio fuera llevado a cabo por diversos especialistas, tanto psicólogos como criminólogos, con la finalidad de poder establecer todas aquellas pautas de comportamiento que en un futuro pudieran desembocar en posibles acciones delictivas.

En este sentido, el citado estudio debería ceñirse a estudiar las historias de vida de cada uno de los sujetos que presentan TDAH durante la etapa infantil, centrando el objeto de análisis en el entorno social en el que el individuo haya desarrollado su crecimiento, ya que sus acciones delictivas pueden haber sido el resultado de una mala socialización.

En referencia a dicho contacto perjudicial con el resto de la sociedad por parte del sujeto que presenta este tipo de trastorno, también resulta preciso señalar la acción nega- 
tiva que ejercen los dispositivos neuronales intervinientes en el desarrollo del TDAH en determinadas personas. En este sentido, surge la idea de proceder a un estudio exhaustivo del comportamiento delictivo de dichos individuos, con la intención de analizar una posible correlación que pueda llegar a considerarlos como sujetos inimputables.

Ante la posible incidencia de la inimputabilidad en sujetos portadores de TDAH, conviene destacar que resultaría difícil de comprender que la presencia de dicho trastorno llevara aparejada tal circunstancia penal. En este sentido, considero que para que se produzca tal acontecimiento, el sujeto debería presentar alguna anomalía que pudiera desembocar en una falta de comprensión de las posibles acciones delictivas.

Para llevar a cabo un estudio pormenorizado de este tipo de sujetos, considero indispensable que las sentencias condenatorias muestren la posible comorbilidad que presentan dichos individuos. Así, se podría analizar si las personas que presentan TDAH y han cometido alguna acción delictiva padecen algún tipo de trastorno o enfermedad que les haya influido de forma imperante en la comisión de dicho delito. En este sentido, por ejemplo, se podría investigar si el consumo de determinadas sustancias podría desencadenar en estas personas la comisión de alguna infracción penal.

En referencia a la relación existente entre la presencia de TDAH y las características de los diversos delitos cometidos, el estudio de las sentencias condenatorias muestra cómo serán las acciones cometidas dentro del ámbito familiar y las realzadas contra la libertad sexual las que presentarán unos porcentajes más elevados. En este sentido, considero fundamental la realización de futuras investigaciones en las que se pueda estudiar la posible correlación entre la comisión de dichas acciones con la presencia de una manifiesta pluralidad de trastornos en el individuo infractor.

Finalmente, al igual que sucede en el estudio desarrollado en España ${ }^{37}$, tras el análisis de las diversas sentencias encontradas, se puede apreciar como el TDAH no puede ser considerado como un trastorno productor de violencia, siendo necesario que existan otras alteraciones o concurran contextos violentos que predispongan a dichas personas para que puedan cometer las acciones delictivas.

\section{JURISPRUDENCIA}

- Sentenze Cassazione Penale, sezione III., n. 4.905, [17/05/1985].

- Sentenze Cassazione Penale, sezione VI., n. 13.897, [11/02/2010].

- Sentenze Cassazione Penale, sezione III., n. 41.702, [13/06/2012].

37 PINTADO ALCÁZAR, A., «Análisis criminológico... cit., pág. 184. 
- Sentenze Cassazione Penale, sezione III., n. 28.229, [18/04/2013].

- Sentenze Cassazione Penale, sezione III., n. 19.424, [07/02/2014].

- Sentenze Cassazione Penale, sezione III., n. 20.419, [07/02/2014].

- Sentenze Cassazione Penale, sezione V., n. 36.216, [08/04/2015].

- Sentenze Cassazione Penale, sezione V., n. 46.450, [06/05/2015].

- Sentenze Cassazione Penale, sezione III., n. 49.165, [06/10/2015].

- Sentenze Cassazione Penale, sezione III., n. 50.752, [14/09/2016].

- Sentenze Cassazione Penale, sezione VI., n. 41.128, [27/09/2016].

- Sentenze Cassazione Penale, sezione IV., n. 43.864, [06/10/2016].

- Sentenze Cassazione Penale, sezione VI., n. 10.906, [15/02/2017].

- Sentenze Cassazione Penale, sezione I., n. 23.385, [28/04/2017].

- Sentenze Cassazione Penale, sezione VI., n. 38.993, [06/06/2017].

- Sentenze Cassazione Penale, sezione VI., n. 25.248, [03/05/2018].

\section{BIBLIOGRAFÍA}

AGUILAR CÁCERLES, M., El trastorno por déficit de atención e hiperactividad (TDAH). Aspectos jurídico-penales, psicológicos y criminológicos, Madrid, 2014.

AMERICAN PSYCHIATRIC ASSOCIATION (APA), Diagnostic Statistical Manual of Mental Disorders, Second Edition (DSM-II), Washington D.C., 1968.

BORRELLI, G., «Dei delitti contro l'ordine pubblico», en Lattanzi, G., y Lupo, E., Codice Penale. Rassegna di giurisprudenza e di dottrina, Vol. IX, Milán, 2015.

CHESS, S., «Diagnosis and treatment of the hyperactive child», en New York State Journal of Medicine, Vol. 60, 1960.

CIANCIOLA, G., Genere e crimine nella società postmoderna. La donna Kamikaze, Roma, 2009.

COLOMBO, A., «Gli omicidi Italia. Tendenze e caratteristiche dall’Unità a oggi», en Rassegna Italiana di Criminologia, Vol. 4, 2011.

DE AMICIS, G., «Dei delitti contro la fede pubblica», en Lattanzi, G., y Lupo, E., Codice

Penale. Rassegna di giurisprudenza e di dottrina, Vol. VI, Milán, 2015.

DELPINO, L., y PEZZANO, R., Manuale di Diritto Penale. Parte Speciale, Nápoles, 2018. 
HALL, C.L., NEWELL, K., TAYLOR, J., SAYAL, K., SWIFT, K.D., and HOLLIS, C., «"Mind the gap" - Mapping services for young people with ADHD transitioning from child to adult mental health services», en BMC Psychiatry, Vol. 13(186), 2013.

LÓPEZ SOLER, C., CASTRO SÁEZ, M., BELCHÍ, A.I., y ROMERO MEDINA, A., «Descripción clínica: el trastorno por déficit de atención e hiperactividad y los trastornos del comportamiento», en López Soler, C., y Romero Medina, A., TDAH y trastornos del comportamiento en la infancia y la adolescencia, Madrid, 2013.

MORILLAS FERNÁNDEZ, D.L., «Análisis de las principales variables de la delincuencia juvenil en España», en Revista de Derecho, Empresa y Sociedad, REDS, Vol. 3, Época I, 2013.

ODETTE, E., «Potenziamento umano e dirieto penale (*) Il «caso» dell'enhancement cognitivo», en Rivista italiana di diritto e procedura penale, Vol. 55(3), 2012.

PALERMO, G.B., y MASTRONARDI, V., «L'omicidio. Profili comparatistici Italia-USA», en Supplemento alla Rivista di Psichiatria, Vol. 47(4), 2012.

PINTADO ALCÁZAR, A., «Análisis criminológico de la relación TDAH/delincuencia en España», en Peris Riera, J., El trastorno por déficit de atención e hiperactividad y su repercusión en la responsabilidad penal, Madrid, 2017.

REALE, L., and BONATI, M., «ADHD prevalence estimates in Italian children and adolescents: a methodological issue», en Italian Journal of Pediatrics, Vol. 44(1), 2018.

REALE, L., COSTANTINO, M.A., SEQUI, M., and BONATI, M., «Transition to adult mental health services for young people with ADHD», en Journal of Attention Disorders, Vol. 22(6), 2018.

ROMANO, B., FINAZZO, S., MANNO, M.A., y MINO, A., «Reati contro la persona», en Grosso, C.F., Padovani, T., y Pagliaro, A (Dirs.)., Trattato di diritto penale, Vol. XIV., Milán, 2016.

ROSE, S., Il cervello del ventunesimo secolo. Spiegare, curare e manipolare la mente, Roma, 2007.

TIMMI, S., TAYLOR, E., CANNON, M., McKENZIE, K., and SIMS, A., «ADHD is best understood as a cultural construct», en The British Journal of Psychiatry, Vol. 184(1), 2004.

TRINCI, A., y FARINI, S., Compendio di Diritto Penale. Parte Speciale, Roma, 2018.

ZINGIRIAN, G., La danza delle circostanze: Profili applicativi degli accidentalia delicti, Vicalvi, 2015. 